\title{
New educational tools and curriculum enhancements for motivating engineering students to design and realize bio-inspired products
}

\author{
H. A. Bruck, A. L. Gershon, I. Golden, S. K. Gupta, \\ L. S. Gyger Jr., E. B. Magrab \& B. W. Spranklin \\ Department of Mechanical Engineering, University of Maryland, \\ College Park, MD, USA
}

\begin{abstract}
The use of bio-inspiration to create new products and devices requires the development of new design tools and manufacturing technologies, as well as the education of students capable of using them. At the University of Maryland, we have developed new educational tools that emphasize bio-inspired product realization. These tools include the development of a bio-inspired design repository, a concurrent fabrication and assembly technology that focuses on the use of multi-piece, multi-stage molds to affordably manufacture large numbers of bio-inspired products, and a series of undergraduate curriculum enhancements. This paper provides an overview of these two technologies and describes how these technologies are integrated into the Mechanical Engineering curriculum. The new educational tools provide students with the fundamental design and manufacturing principles needed in bio-inspired product and device development. The curriculum changes culminate in an elective course undertaken in their senior year.

Keywords: bio-inspired design and manufacturing, design repository, concurrent fabrication and assembly, multi-piece multi-stage molding, mechanical engineering curriculum.
\end{abstract}

\section{Introduction}

For millions of years, nature has been creating materials, plants, insects, and animals that are optimized in many regards. These materials and creatures have 
evolved in ways that enable them to sustain their existence in variety of ways. One example is the common housefly, shown below in Figure 1. It has wings that are extremely thin, with minimal reinforcement, allowing for high speed oscillations, resulting in high efficiency and speed. Another example of a creature that nature has optimized is the eye of the golden eagle, which has a shape and size that enables it to detect prey from high places. Some creatures, such as the chameleon, optimize their survivability by changing color to camouflage them from predators in a variety of environments.

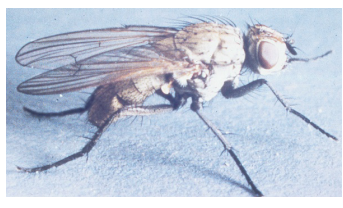

(a)

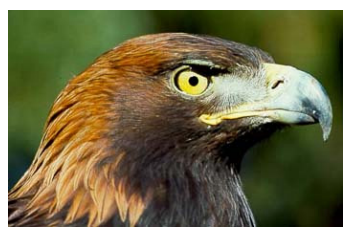

(b)

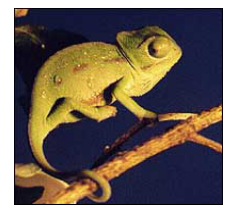

(c)

Figure 1: Natural Designs: (a) house fly; (b) golden eagle; (c) chameleon.

Engineers are frequently asked to design new materials, structures, mechanisms, and processes. To design these new products and artifacts often requires a search for new ideas. One source for new ideas can be found in nature. By learning about the way plants and animals have been created and function in nature through millions of years of evolution, engineers gain new inspiration to develop new products, which has been termed "bio-inspired" or "biologicallyinspired" products. Many existing design tasks can also be re-examined to find new solutions using nature as a tool for inspiration. Unfortunately, many engineers may not think to use nature as a source of inspiration because most undergraduate engineering curriculums do not adequately emphasize its importance. It is our belief that if engineers are educated on the ways nature handles certain tasks, they will in turn be able to apply new concepts to future designs.

Bio-inspired design has recently been explored by many researchers, and this has had very positive outcomes for robotics, aerodynamic systems, biomedical devices, materials, and computational systems. The materials community has gained much knowledge from nature to develop better microstructures for materials, and several books have been published dealing with the natural materials themselves as well as synthetic replicas, or biomimetic materials ([1], [2], and [3]). The robotics community has also turned to nature for inspiration and published books ([4] and [5]) that have helped designers develop robots that use animal-like gaits rather than wheels for better functionality and efficiency to meet special requirements. Structural engineering researchers have also been studying nature for new ideas on how geometry affects load handling ability and how it can be sensed ([6], [7], and [8]) to create stronger and more adaptive structures. Success has already been realized using bio-inspired concepts, and it is our aim to educate undergraduate students on these recent advances in bioinspired design so that they may be able to use ideas taken from nature and apply them to future designs. 
Slowly, bio-inspired concepts have been implemented into a variety of products. The reason for this slow growth has been two challenges that need to be overcome for students to learn about and discover the full potential of nature's designs. The first challenge is to make students more aware of biological concepts and bio-inspired products. Currently, many engineers overlook solutions found in nature simply because they do not know how to look there and there is no repository of bio-inspired solutions that they can explore. To address this challenge, we are developing a searchable and expandable bio-inspired design repository that complements another approach being developed at The Center for Biomimetic and Natural Technologies at the University of Bath that focuses on integrating biology into an indexing method for design retrieval, known as TRIZ, by introducing biomimetic design concepts [9]. The second major challenge comes from the fact that most biological systems are geometrically complex and are highly heterogeneous and use variation in the material properties to achieve improved material-function compatibility. Furthermore, to achieve high degree of fault tolerance and adaptive behavior, they make use of sensors and actuators distributed throughout the system. Traditional manufacturing processes are not able to easily manufacture bioinspired designs based on these characteristics. To address this challenge, we have developed a new multi-piece multi-stage molding processes that is part of a new class of manufacturing technologies that permit concurrent fabrication and assembly for realizing bio-inspired designs.

While the design repository and concurrent fabrication and assembly manufacturing technology are needed in order for students to learn about designing and realizing bio-inspired products, it will be difficult for them to properly utilize these tools without introducing the appropriate design, manufacturing, and materials subjects into the curriculum. These teaching materials must provide students with an understanding of the motivation behind bio-inspired products, an understanding of what has already been achieved, and a knowledge of the core mathematical and scientific principles behind the realization of bio-inspired products that have been achieved through design and manufacturing.

This paper will now describe educational activities being pursued in the Department of Mechanical Engineering at the University of Maryland to meet these challenges through new educational tools and curriculum. The next section will introduce the approach and techniques that were used to create a new repository of bio-inspired products and concepts, a new multi-piece multi-stage molding technology for manufacturing of bio-inspired products with no assembly, and a new undergraduate mechanical engineering curriculum for bioinspired product realization.

\section{Development of new bio-inspired design repository}

In developing a repository of bio-inspired products and concepts, it is important to include information about the biological systems, as seen in the biological effects database, while also presenting information on product development that 
will aid engineers. When creating a functional language to access this information, a functional basis has proven to aid in archiving and retrieving the information. However, it is also important to allow the user freedom to express the functions in terms of language and sentence structure. Whatever terms are used should be common language terms and not arranged in an arbitrary hierarchy. The natural language analysis has also shown that by searching for many alternatives to a single function term, many useful results may be found. Lastly, easy to use and flexible search tools are required to locate meaningful results.

The complete system has three parts: (a) Repository of bio-inspired products and concepts, (b) Functional Description Template, and (c) Search tools. The interaction of these three elements is shown in Figure 2. In order to add a product or concept into the repository, the user must fill out the data entry form. The entry may often be made by a biologist adding concepts from biological research or by an engineer archiving a bio-inspired product. In either case, the Functional Description Template is used to record the functions of the product or biological system. Following this process, the entry is stored. A user may then use a search form to locate information on bio-inspired work. The products or concepts that are returned can then be applied to the design to create a new bioinspired design. This design can then be added into the repository as a new bioinspired product.

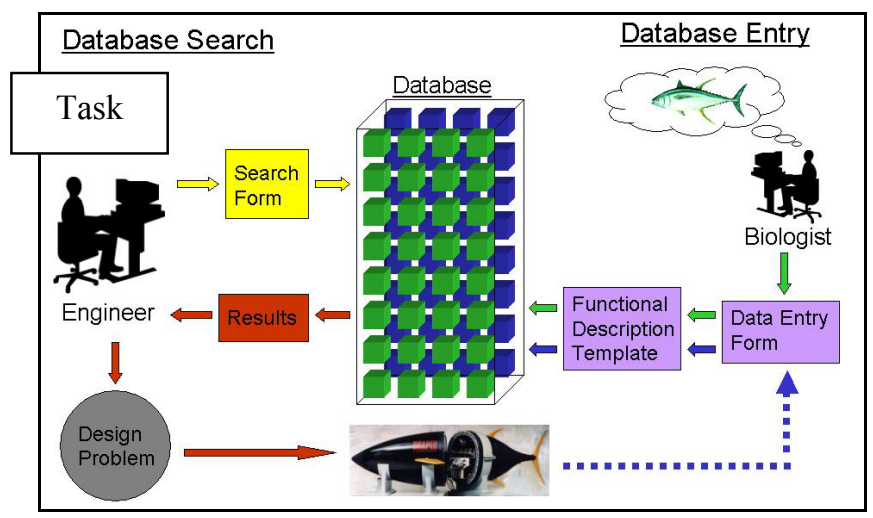

Figure 2: $\quad$ System overview.

\subsection{Contents of repository}

The repository being developed archives bio-inspired products and concepts. Bio-inspired products refer to products in any stage of development that have taken their inspiration from nature. These products may be anything from a new material substance to a complex mechanical device; they do not necessarily have to be consumer products. A bio-inspired concept refers to an observation of some function being met in nature. This concept need not be implemented into a product yet. Such concepts may be archived into the repository and any potential 
applications may be recorded. Currently, the repository contains 85 records of bio-inspired products. These products were identified and researched though a search of journal papers, conference papers, magazine articles, and websites that discuss current bio-inspired research. The searches that were conducted explored bio-inspired actuators, sensors, materials, robots, and various other mechanical devices and consumer products that were inspired by nature. All of the products that were located were then recorded into the repository. For a bio-inspired product, all of the above fields may be populated. If a bio-inspired concept is being archived, the product information fields will remain empty, except possibly the applications field. The product function, or in the case of a concept the function of the biological system, requires many fields that are populated by using the Functional Description Template.

\subsection{Functional Description Template}

In order to develop an easy to use and expressive method for recording functions, it was necessary to first understand how people naturally express a functional statement. To accomplish this, an informal study was done asking people to write down the functions of common products. From these results, it was clear that there was no uniform way to represent a function. Some functions were very brief statements, while others were lengthy descriptions. However, all the functional statements used the same elements to form a sentence (verbs, nouns, etc.) Therefore, if common arrangements of these terms could be identified, then standardized templates could be provided for users and the desired terms could be entered. This concept is the basis for the Functional Description Template, a new method for recording product functions in our design repository that provides a predetermined sentence structure as a template to allow the user to form more complete functional descriptions. The methods of data entry used to fill in the template are a combination of freeform text entry and menu selection, which allow the recorded information to be efficiently archived and retrieved while ensuring that the system is easy to use by both engineers and biologists. In our design repository, the template makes use of the following three primary terms: (a) Action, (b) Entity, and (c) Property. Prepositions and conjunctions are also available. Adjectives and adverbs, however, are currently not used in the Functional Description Template. This omission is due to the fact that such terms were found to be non-essential to describing a function and merely offer additional information. However, the addition of these terms to the template could be completed at a later time.

\subsection{Performing search on the repository}

It is essential that the information stored in the repository be easily accessible to users in a straightforward manner and must provide meaningful results. To accomplish this, we have made the following three search tools available: (a) keyword search, (b) category filter, and (c) function search. In the keyword search a user enters the desired search terms using freeform text entry. fields will be searched. The search for our bio-inspired design repository is conducted by using a string matching algorithm that is a combination of two other string 
matching algorithms; namely, equivalence method and similarity method. For the category filter, results are filtered based upon the fields that are recorded using menu selection. These fields include: (a) Product Type, (b) Biological Type, and (c) Development Stage. The final search tool is the function search, which is used to specifically search for products or concepts that meet a particular function or set of functions. While the keyword search may be used to search the functions of the products and concepts in the repository, the function search is specifically designed to search by function. This search tool aids the user in creating the functional statements for which to search and conducts a more flexible search that returns more useful results.

\section{Product Information}

Product Name: VCUUV

Product Type: Mechanism: Robot

Product Description: An unmanned underwater vehicle with a low drag body and flexible hull. Uses vorticity control for both propulsion and maneuvering, and caudal and pectoral fins for steering.

Development Stage: Product development

Applications: Mine reconnaissance, surveying, cable laying

\section{Functions}

- $\quad$ Propel robot through water

- Detect position of mines

- Measure volume of mines

- Regulate linear velocity of robot

- Regulate angular velocity of robot

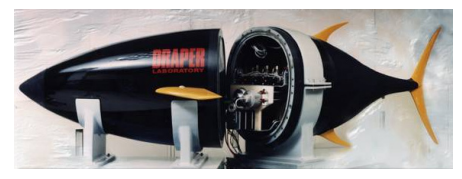

\section{Biological Inspiration}

Biological Name: Yellow fin Tuna

Biological Type: Animal: Fish

Biological Description: The tuna has a very streamlined body and is highly maneuverable. It can accelerate and decelerate rapidly and maneuver in tight space

\section{Resources}

Primary Source: J.M. Anderson and P.A. Kerrebrock, "The Vorticity Control Unmanned Undersea Vehicle (VCUUV): Performance Results," $11^{\text {th }}$ International Symposium on Unmanned Untethered Submersible Technology, Draper Report No. P-3747, Durham, NH, August 1999.

Additional References: http://www.draper.com

Figure 3: $\quad$ Example of record from search of bio-inspired design repository.

\subsection{Example}

Consider a scenario in which a student is designing an automated vehicle that can be used for underwater surveillance. The student, familiar with the recent advances in bio-inspired engineering, decides to search the repository of bio- 
inspired products and concepts. The student may search for ideas using a combination of the search tools described above. In this case, a search is conducted for the keywords "underwater reconnaissance", as well as the function "move device in liquid". From this search, many results are returned, some of which contain only the keyword "underwater" and some of which contain a variation of the function "move device in liquid". However, the top result is one that describes an unmanned underwater vehicle that was inspired by the tuna [10]. This entry in the repository contains the keyword "underwater" in the product description and contains the keyword "reconnaissance" in the applications. Furthermore, one of the product's functions, "propel robot through water", closely matched the search function. The student may then view the complete record, as shown in Figure 3.

\section{Multi-piece multi-stage molding for bio-inspired product}

For students to implement designs such as the one described in the case study, a new Concurrent Fabrication and Assembly technology based on Multi-Piece Multi-Stage Molding process was developed at the University of Maryland to affordably manufacture large quantities of bio-inspired products. This technology is focused around the design and manufacturing of the molds. Designing the mold for bio-inspired products is a very challenging task, because the complexity of the material distributions and geometries are not conducive to generating the single parting direction required for a standard two-piece mold. In addition, complex molds often require very complex undercuts to realize the entire structure. Multi-piece molds overcome these restrictions by having many parting directions. These molds have more than one primary parting surface, and consist of more than two mold pieces or sub-assemblies. Each of these mold pieces will have a different parting direction. The freedom to remove the mold pieces from many different directions eliminates the undercuts produced by twopiece molds. A multi-piece mold can be visualized as a 3D jigsaw puzzle, where all the mold pieces fit together to form a cavity and then can be disassembled to eject the molded part. Moreover, since there are no actuated side cores in multipiece molds, the tooling cost is significantly lower. This makes multi-piece molding technology an ideal candidate for making geometrically complex objects. The multi-piece mold can be enhanced through multi-stage molding, where different material sections are injected in sequence into a mold or set of molds. This enables fully assembled components to be fabricated 'in-mold', resulting in concurrent fabrication and assembly.

The concurrent fabrication and assembly of components using the multi-piece multi-stage molding process allows one to fabricate heterogeneous structures with material interfaces that are geometrically complex and exhibit superior fracture and fatigue behavior, as well as improved functional performance [11, 12]. Specifically, multi-piece multi-stage molds can create multi-material objects to improve material-function compatibility for the overall object, and to embed prefabricated sensors/actuators during the molding process to eliminate the need for post-molding assembly and to significantly improve reliability. It is also 
more scalable, simpler, faster, and cheaper than conventional manufacturing processes.

An example of a bio-inspired design that was manufactured using this technology is a snake robot module. A solid model of the module is shown in Figure 4. The mechanical structure of the module was assembled entirely inmold, and consists of a universal joint, along with docking connectors. The male and female connectors are located at opposite ends of the structure, and are geometrically correct, although a more flexible material must be used in order for them to be functional (something that can easily be done with a different grade of polyurethane). The module was assembled via a two-stage transfer molding process, where a second material was used to make the universal joint. The entire module consisted of only three parts, the two on either side of the joint, and the cross-shaped piece making the joint; consequently, no additional fasteners or shafts are required. The concave interior feature of the female side of the docking mechanism was created using a split-core technique. An assembled snake robot can be seen in Figure 5.

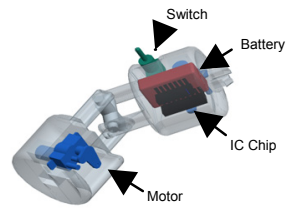

Figure 4: Solid model of snake robot module.

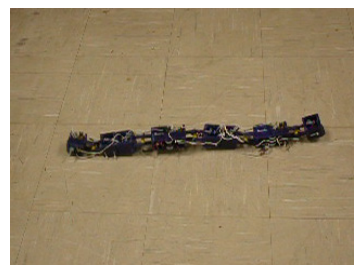

Figure 5: Assembled snake robot.

\section{Curriculum for bio-inspired product realization}

To realize bio-inspired products using the new design tool and manufacturing technology, a new undergraduate curriculum for mechanical engineers was developed and implemented at the University of Maryland [13]. This curriculum involves several single-lecture modules that cover a broad range of topics in bioinspired design, manufacturing, and materials that are implemented in several core courses, as well as some senior electives. The courses and modules developed for these courses are as follows:

Introduction to Design - Introduction to bio-inspired design

Controls and Robotics - Bio-inspired design of mobile robots

Controls and Robotics - Bio-inspired artificial muscles

Measurement and Instrumentation - Bio-inspired sensors

Manufacturing Processes - Bio-inspired concurrent fabrication and assembly

Manufacturing Processes - Bio-inspired self-assembly

Introduction to Materials - Bio-inspired functionally graded materials

Descriptions of these modules can be found at www.bioinspired.umd.edu, and are available in PDF format by emailing a request to the authors. In addition to 
these modules, a new senior elective was developed for Bio-inspired Product Realization to provide more content to these modules, as well as a design project resulting in bio-inspired products as the Snake Robot in Figure 6. An assessment of one of the manufacturing modules and the materials module were also undertaken to determine the efficacy of the curriculum. These assessment results indicated that a large majority of the students had very little prior knowledge of bio-inspired products and expressed strong interest in the subject. In addition, they indicated that they were able to acquire introductory knowledge from the modules.

\section{Conclusions}

The work presented in this paper describes three distinct contributions to motivate engineering students to use bio-inspiration to design and realize products. (1) A design repository of bio-inspired products and concepts based on a Functional Description Template was developed which contains detailed information about existing products in various stages of development and biological systems that provides new design concepts to students. (2) A concurrent fabrication and assembly technique for manufacturing products from the bio-inspired designs using a new multi-stage multi-piece molding technology for manufacturing products from bio-inspired designs that solves many of the mold design issues for complex material distributions and geometries. (3) A new curriculum for bio-inspired product realization to educate and train undergraduate mechanical engineering students to include bio-inspired ideas in their products using the new design tool and the new manufacturing technology.

Our new developed tools should also provide benefits to the engineering community at large. The repository, when populated with more and more products and concepts, will make knowledge of bio-inspired ideas more accessible to engineers. This information can lead to the development of new and innovative products that may provide improved product performance and lower cost devices. Additionally, the repository and functional description template are not limited to archiving bio-inspired products and concepts. In time, the repository could be used to archive any product or design concept and offer a more complete knowledge base for engineers seeking candidate design concepts.

\section{Acknowledgement}

This worked was supported by NSF grant EEC0315425. Opinions expressed in this paper are those of the authors and do not necessarily reflect opinions of the sponsors.

\section{References}

[1] S. Mann, ed. Biomimetic materials chemistry. New York: VCH, 1996. 
[2] M. Sarikaya and I.A. Aksay, eds. Biomimetics: design and processing of materials. Woodbury, N.Y.: AIP Press, 1995.

[3] R.P. Wool and X.S. Sun. Bio-based polymers and composites. Boston: Elsevier Academic Press, 2005.

[4] R.J. Duro, J. Santos, and M. Graña, eds. Biologically inspired robot behavior engineering. New York: Physica-Verlag, 2003.

[5] S. Hirose. Biologically inspired robots: snake-like locomotors and manipulators. New York: Oxford University Press, 1993.

[6] A. Bejan. Shape and structure, from engineering to nature. New York: Cambridge University Press, 2000.

[7] C. Mattheck. Design in nature: learning from trees. New York: SpringerVerlag, 1998.

[8] A. Skordos, P.H. Chan, J.F.V. Vincent, and G. Jeronimidis. "A Novel Strain Sensor Based on the Campaniform Sensillum of Insects". Phil. Trans. of the Royal Society London A, Vol 360, pp 239 - 253, 2002.

[9] J.F.V. Vincent and D.L. Mann. "Systematic Technology Transfer from Biology to Engineering". Philosophical Transactions of the Royal Society: Physical Sciences, Vol 360, pp 159-173, 2002.

[10] J.M. Anderson and P.A. Kerrebrock "The Vorticity Control Unmanned Undersea Vehicle (VCUUV): Performance Results". $11^{\text {th }}$ International Symposium on Unmanned Untethered Submersible Technology, Draper Report No. P-3747, Durham, NH, August, 1999.

[11] H.A. Bruck, G. Fowler, S.K. Gupta, and T.M. Valentine. "Towards bioinspired interfaces: Using geometric complexity to enhance the interfacial strengths of heterogeneous structures fabricated in a multi-stage multipiece molding process". Experimental Mechanics, Vol 44, pp 261-271, 2004.

[12] R.M. Gouker, S.K. Gupta, H.A. Bruck, and T. Holzchuh. "Manufacturing Of Multi-Material Compliant Mechanisms Using Multi-Material Molding". to appear in International Journal of Advanced Manufacturing Technology, 2006.

[13] H.A. Bruck, A.L. Gershon, and S.K. Gupta. "Enhancement of Mechanical Engineering Curriculum to Introduce Manufacturing Techniques and Principles for Bio-inspired Product Development”. Proceedings of the ASME International Mechanical Engineering Congress and Exposition, pp 1-6, 2004. 